étroites, toutes chosse restanl égales d'ailleurs, et que le débit linéaire varierait donc dans le même sens que la distance admise entre piles; c'est ce que tendraient à indiquer les formules de débit des barrages à contractions latérales. L'expérience a fourni un résultat opposé, et les courbes de la figure 2 montrent la valeur bien supérieure du débit linéaire, pour une charge donnée, dans le cas d'une largeur de passe de 20 mètres, vis-à-vis d'une largeur de passe de 67 mètres. Ce résultat, à priori surprenant, peut aisément s'expliquer : il suffit de considérer les figures 3 et 4 pour voir la différence énorme des valeurs absolues des contractions existant dans l'un et l'autre cas; la longueur des piles étant d'une trentaine de mètres, il se produit, dans le cas de la passe de 20 mètres de largeur, un phénomène analogue à celui qui caractérise l'écoulement dans un ajutage cylindrique, les filets, après une contraction initiale, rejoignent les parois latérales des piles de telle sorte que l'extrémité aval de la passe débite sensiblement à pleine section; au contraire, dans le cas de la passe de 67 mètres de largeur, ce phénomène ne se produit pas, les piles, en contact avec une zone d'eau tourbillonnaire, n'intéressent que par leur extrémité antérieure la zone d'écoulement direct dont l'aspect rappelle la coupe d'un écoulement par orifice en mince paroi. Ce fait explique le phénomène constaté ; il est même curieux de remarquer que le rapport des débits linéaires correspondant aux deux cas envisagés est du même ordre de grandeur, en moyenne, que le rapport des coefficients de débit de l'ajutage cylindrique et de l'orifice $\in n$ mince paroi ; il ne s'agit là, d'ailleurs, que d'une analogie, le mouvement considéré ne constituant nullement un écoulement de révolution.

\title{
Technique et économie dans les conduites forcées à diamètre constant et à diamètres variables
}

\author{
par Dott. Ing. Remo Catani
}

\section{I. - Poids des conduites en acier}

Les résultats des publications postérieures à ma première étude en matière ont confirmé en général les résultats que j'avais avancés.

L'importance des conduites en acier est allée toujours en croissant, soit à cause de la valeur plus grande de sa quotepart par rapport à la dépense totale des installations hydroélectriques, soit à cause des hauteurs de chute toujours plus élevées que l'on exploite.

Dans ma première publication, je citais une chute maxima de $950 \mathrm{~m}$. ; on utilise actuellement des chutes de $1.750 \mathrm{~m}$., exemple l'installation de la Dixence et l'on prévoit d'utiliser des chutes de $2.500 \mathrm{~m}$. Les frais d'une conduite à forte pression, dans les conditions actuelles, se montent à plusieurs millions de lires, une économie de quelques unités au pour cent du coût total représente des fractions respectables d? million.

Les propositions et les conclusions de ma première étude furent les suivantes : Une conduite en acier au lieu d'avoir un diamètre constanl devrait avoir des diamètres décroissants vers le bas en subdivisant la conduite en un certain nombre de tronçons de longueur égale, mais en attribuant à chacun d'eux une perte croissante, suivant des nombres naturels, tout en laissant constante la perte totale de charge $\mathrm{Y}$; on réduit ainsi de $5 \%$ le poids de la conduite, on augmente le volume de l'eau d'écoulement dans celle-ci, on diminue sa force vive et le débit maximum de la conduite en cas de rupture dans la partie à pression maximum, ainsi que l'épaisseur maximum et le diamètre des tronęons inférieurs. Ces conceptions développées en son temps doivent être appliquées avec sagacité à de nombreux. et très différents cas de la pratique.

Généralement, l'axe d'une conduite se développe en profil dans un plan vertical même lorsque le tracé comporte des angles planimétriques. A la suite des déformatio s, souvent considérables, l'axe de la conduite peut présenter des tronçons curvilignes et les parties rectilignes avoir des inclinaisons variables. (de 0 à $90^{\circ}$ ). A l'extrémité aval de la conduite, généralement horizontale, il faut ajouter les longueurs virtuelles des dérivations. Ces longueurs, comme il est indiqué dans ma dernière note, s'obtiennent en réduisant les longueurs réelles des dérivaArticle published by SHF and available at nttp:I/Tww.Sht-Ihb.org or tions dans le rapport des carrés des diamètres de chacune d'elles et du diamètre du tronçon faisant office de collecteur d'où elles se détachent. D'autre part, la conduite peut avoir une section circulaire constante, ou bien la variation de la section peut être continue ou discontinue.

Enfin, les conduites forcées en acier peuvent être rivées, soudées, frettées, sans soudures (Mannesmann ou laminées) et dans la même canalisation, on peut retrouver ces divers types

Ce qui précède met en évidence la variété possible dans les installations industrielles des conduites forcées, la nécessité d'une étude préalable et particulière à chaque cas, ainsi que l'impossibilité d'émettre une théorie générale directement applicable à tous les cas de la pratique. Chaque fois qu'il a été fait un essai dans ce sens, on a constaté que les conclusions théoriques obtenues devaient invariablement être modifiées à leur application dans chaque cas bien déterminé. Il suffit de citer, à cet effet, les difficultés rencontrées pour déterminer les équations des courbes éventuelles dans le profil du tracé. En pratique, la solution des-problèmes de chaque canalisation est obtenue à la suite d'études analytiques et graphiques particulières à chaque cas et dont la méthode ainsi que ses difficultés dépendent non seulement de la complexité de la canalisation, mais de toute l'installation hydro-électrique.

Ces considérations m'on déterminé à développer en entier, dans ma première Notice, un exemple pratique (fig. 1) qui sera textuellement repris dans la présente.

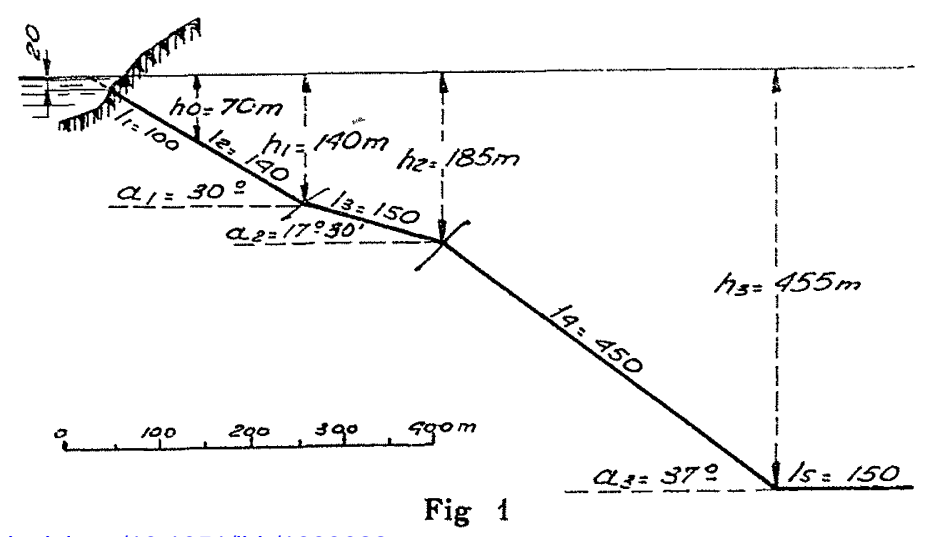


A) Diamètre constant. - Pour une conduite à diamètre constant, on avait établi les relations sous-indiquées pour la détermination" des poids en kilog., dans lesquelles $h$ représente la charge d'un tronçon quelconque, $H$ et $D$ la chute et le diamètre; toutes les longueurs seront exprimées en mètres :

$$
\begin{array}{ll}
p=2 h d^{2} & \text { par mètre linéaire } \\
P=H^{2} D^{2} & \text { canalisation verticale } \\
P=\frac{H^{2} D^{2}}{\sin \alpha} & -\quad \begin{array}{c}
\text { inclinée de } x^{0} \text { sur l'horizon- } \\
\text { tale }
\end{array} \\
P=2 H L D^{2} & -\quad d^{\prime} \text { tane longueur de } L \text { mètres. }
\end{array}
$$

Pour le premier troncon où l'épaisseur est indépendante de la pression, le poids doit être déterminé d'après la relation (1). L'épaisseur de la conduite peut être exprimée en centimètres par le chiffre $5 D$ ( $D$ diamètre de la conduite en mètres).

Bien qu'en général l'épaisseur $e$ d'une conduite en acier soit exprimée par l'équation :

$$
e=0,714 h d
$$

pour le premier tronçon de la conduite on prendra comme il a été dit :

$$
e=5 d
$$

De l'égalité de ces deux équations qui expriment la valeur de $e$, on déduit que l'épaisseur restera constante jusqu'à une profondeur de $70 \mathrm{~m}$. environ. Le poids du premier tronçon sera, de ce fait, exprimé par :

$$
\mathrm{P}=140 d^{9} l
$$

où $l$ représente la longueur en mètres de la conduite considérée, mème lorsqu'elle est entièrement développée sur une horizontale.

La première dénivellation, si elle comprend tout le tronçon initial, aura un poids donné par l'expression :

$$
\mathrm{P}_{1}=\mathrm{D}^{2}\left(140 l+\frac{h^{2}-4900}{\sin \alpha}\right)
$$

expression valable, même pour des conduites verticales :

$$
\alpha=90^{\circ} ; \sin \alpha=1
$$

et

$$
\mathrm{P}_{1}^{\prime}=\mathrm{D}^{2}\left(\mathrm{H}^{2}+140 l-4900\right)
$$

L'expression (4) nous fournit le poids d'un tronçon rectiligne quelcorque de longueur $L$, si on remplace la hauteur de chute $H$ par la pression hydrostatique $H m$ sur le centre de l'axe du tronçon.

La formule ;

$$
\mathrm{P}=2 \mathrm{H}_{\mathrm{m}} \mathrm{L}, \mathrm{D}^{2}
$$

englobe toutes celles citées précédemment, comme il est d'ailleurs très aisé de s'en rendre compte; elle s'applique aussi lorsqu'il $s^{\prime}$ agit d'un tronçon quelconque $r$ dont l'axe est contenu dans un plan perpendiculaire à la surface de niveau et incliné d'un angle $\alpha_{\mathrm{r}}$ quelconque par rapport à cette dernière (fig. 2).

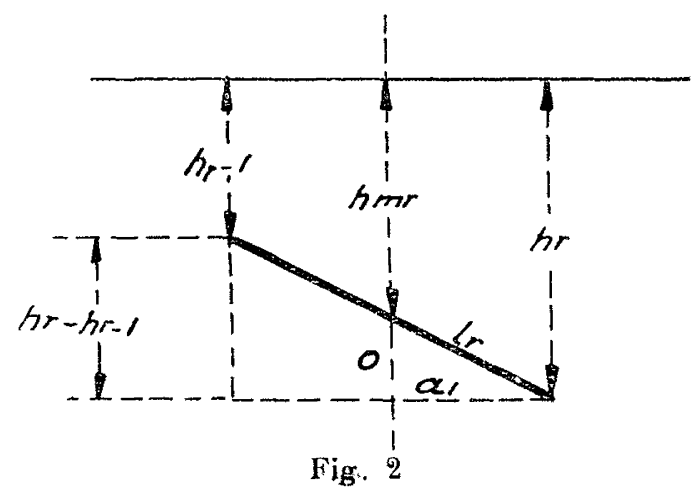

En effet, d'aprìs la relation (3):

$$
\operatorname{Pr}=\frac{\mathrm{D}^{2}}{\sin \alpha_{\mathrm{r}}}\left(h_{\mathrm{r}}^{2}-h_{\mathrm{r}-1}^{2}\right)
$$

mais

et

$$
h_{\mathrm{r}}-h_{1-1}=l_{\mathrm{r}} \sin x
$$

d'où

$$
h_{\mathrm{mr}}=\frac{1}{2}\left(h_{\mathrm{r}}+h_{\mathrm{r}-1}\right)
$$

$$
\begin{gathered}
\mathrm{Pr}_{\mathrm{r}}=2 h_{\mathrm{mr}} l_{\mathrm{r}} \mathrm{D}^{2}=z_{\mathrm{r}} \mathrm{D}^{2} \\
z_{\mathrm{r}}=\stackrel{9}{=} h_{\mathrm{mr}} l_{\mathrm{r}}
\end{gathered}
$$

Il en résulte que, quelle que soil la rotalion que subil l'axe de la conduite autour de $O$, le poids de la conduite reste inchangé. Autrement dil : Toutes les conduites dont les axes passeront par $O$ auront le même poids si elles ont la même longueur et le mème diamètre.

Les relations particulières qui ont été exposées plus haúl de (1) à (4), ainsi que celle générale (4') seront successivement employées suivant le cas et le but auquel on désire aboutir. Dans le but de contrôler les résultats obtenus, on peut souvent les emplover simultanément.

La plus employée sera certainement la formule générale (d') et surtout dans les chapitres suivants où il en sera domé une interprétation plus claire, tout en considérant les résultats obtenus dans les développements analytiques. Les formules de (1) à (4) trouvent, au contraire, une application pratique dans des calculs approximatifs et préliminaircs. Elles donnent des poids tresexacts tant qu'elles sont applicquées dans les conditions fixées au moment de leur établissement. Pour les taux de travail très élevés que l'on a cerdance à employer actuellement, elles peuvent donner des valeurs légèrement plus élevées des poids réels, ou bien compenser seulement, soit l'excès de poids qui se vérifie toujours dans les tronçons initiaux, que celui provenant des inévitables ajustements des dimensions obtenues qu'on arrondit pour avoir des chiffrés pratiques, aussi bien pour les diamètres des conduites que pour leurs épaisseurs. Pour les grandes chutes, les calculs de contrôle et de comparaison entre les conduites cylindriques et celles légèrement coniques, don: nent des résultats d'exactitude suffisante en pratique.

B) Diamètres variables. - En laissant toujours la perte de charge totale $\mathrm{Y}$ constante et en appliquant la loi que j'ai énoncéer on peut, pour les conduites à diamètres variables, déduire leurs poids à partir de celui correspondant aux conduites à diamètre constant. A cet effet, on se servira de coefficients numériques dont la valeur ne dépend que du nombre $n$ de tronçons qui constituent la conduite à plusieurs diamètres.

En indiquant par 1 le poids d'une conduite verlicale, ot inclinée, à diamẹtre constant (pour laquelle $n=1$ ), les poids des conduites à $2,3, \ldots 33$ diamètres successifs sont ;

Poids des conduites verticales ou inclinées à dlametres variables

\begin{tabular}{|c|c|c|}
\hline \multirow{2}{*}{$\begin{array}{c}\text { Nombre } \\
\text { de } \\
\text { tronçons }\end{array}$} & \multicolumn{2}{|c|}{ Poids } \\
\cline { 2 - 3 } & relatifs & diminutions \% \\
\hline 1 & 1,0000 & 0,00 \\
2 & 0,9483 & 5,17 \\
3 & 0,9446 & 5,54 \\
4 & 0,9441 & 5,59 \\
5 & 0,9436 & 5,64 \\
$\ldots \ldots \ldots \ldots$ & $\ldots \ldots \ldots \ldots \ldots$ \\
33 & 0,9282 & 7,18 \\
\hline
\end{tabular}


Si les $n$ tronçons d'égale longueứ appartiennent en partie à la prenière partie verticale ou inclinée de la conduite et en partic, q tronçons par exemple. à la deuxième partie horizontale de la conduite, la diminution de poids de ces derniers est proportionnclle à $q / n$ et la diminution pour la conduite entière est la moyenne composée des parties envisagées.

Si $q / n=0,20$, le poids relatif de la partie horizontale est 0,82 , de façon que si $n=5, q=1$, pour les 4 premiers tron çons, on a 0,94 ; pour la partie horizontale 0,82 et pour la conduile entière 0,92 .

Ces données ont été obtenues par les calculs et en appliquant les formules exposées dans ma première étude.

On s'aperçoit du faible avantage que l'on a à augmenter trop la valeur de $n$. La valeur minimum de $n$ devrait être égalé à trois : $1^{0}$ tronçon initial à épaisseur constante; $2^{\circ}$ tronçon final, généralement à pression constante; $3^{\circ}$ tronçon intermédiaire.

Les pourcentages de la dernière colonne devront être éventuellement diminués pour tenir compte de la correction du prêtmier tronçon de la premtère dénilvellation.

Cela s'applique à la détermination du poids des conduites légèrement coniques.

Pour ce qui concerne les diamètres, la loi de variation est exprimée par la relation :

$$
d_{\mathrm{r}}=0,87 \mathrm{D}(n+1)^{0,2} r^{-0,2}
$$

ou on indique par :

$D=$ le diamètre de la conduite à section constante et à pèrté de charge équivalente.

$n=$ nombre dè tronçons d'égalè longueur qui constituent la conduite.

$r=$ numéro d'ordre du tronçon considéré.

Les diamètres $d$ décroissent même lorsque la conduite parcourt une partie plate ou en contrepente (installation des Aciéries de Terni, de Nordhausen, etc.) En voulant obtenir des tronçons coniques avec sommet vers le bas de chaque dépression, il faudra procéder comme je l'ai exposé dans ma premiére Notice.

La pression hydrostatique moyenne $h \cdot d u$ tronçon $r$ pour une conduite verticale (sans tharge sur la prise) est exprimée par :

$$
h_{\mathrm{r}}=\frac{\mathrm{H}}{n}\left(r^{2}-\frac{1}{2}\right)=\frac{\left.\mathrm{H}_{1} 2 r-1\right)}{2 n}
$$

il s'en suil que :

$$
h_{\mathrm{r}} d_{\mathrm{r}}^{\tau}=0,19 \mathrm{H} \mathrm{D}^{\dagger} \frac{(n+1)^{14}}{n} \cdot \frac{2 r-1}{r^{14}}
$$

et en pởant :

$$
\begin{aligned}
& (2 r-1) r^{-1,4} \cdots(\bar{r}) \\
& \psi(n)=0,19 \frac{(n+1)^{1,4}}{n}
\end{aligned}
$$

on oblient

d'ou

$$
0,19 \mathrm{H} \mathrm{D}^{7} \frac{(n+1)^{1,4}}{n}=\mathrm{H} \mathrm{D}^{7} \psi(n)=\mathrm{B}
$$

$$
h_{\mathrm{r}} d_{1}^{\dagger}=\mathrm{B} \varphi\left(r^{2}\right)
$$

Oh aboutit à the equation de ce type à la suite des considé rations que nous expóserons plus loin. On pourra aussi faciles menl voir qu'on y est conduit par d'autres procédés analyliques s'inspirant de conceptions puremenl économiques.

Le capital investi pour l'installation d'un mètre courant de la conduite est fonction dirēête de $p$, donné par la relation (1); celui correspondant aux pertes est une fonction inverse de $y$; on peut donc exprimer le capital total $c$ par la relation :

$$
c=f\left(\frac{p}{y}\right)=f\left(\frac{2 h d^{2}}{k Q^{2} d-5}\right)=\frac{2}{k Q^{q}} \cdot f_{1}\left(h d^{\top}\right)
$$

on y aboutit aussi si l'on détermine un coefficient $b$ tel qu'on puisse écrire l'équation suivante :

$$
c=h d^{2}+b d^{-5}
$$

En dérivant, égalant à zéro et en résolvant on obtient :

$$
h d^{\top}=\frac{5}{2} b
$$

d'où :

$$
d=\sqrt[7]{\frac{5}{2} \frac{b}{h}}=1,14 \sqrt[7]{\frac{b}{n}}
$$

Lâ diffé̉refice eñtre ces rèlations et l'équation précédemment établie $h_{\mathrm{r}} d_{\mathrm{r}}^{2}=B \varphi_{i}(r)$ réside surtout dans les fonctions $\psi(n)$ et $\varphi(r)$ qui présentent quelqques particularités.

Dans d'autres formules de cette mêne Notice, le produit

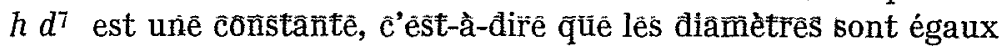
pour une váleur de $h$ donnnée. Il s'en suit que, dans des tronçons d'inclinaisons opposéés, le diamètre diminue et augñente.

Dans l'expression $h d^{7}=B \%(r)$, par contre, la fonction $\because(r)$ induít la constảnte, la diminution du diamètre se vérifie dans le sens de l'écoulement.

Le tableau suivant nous permet de calculer rapidement la valeur de $\bar{B} \varphi(r)$ :

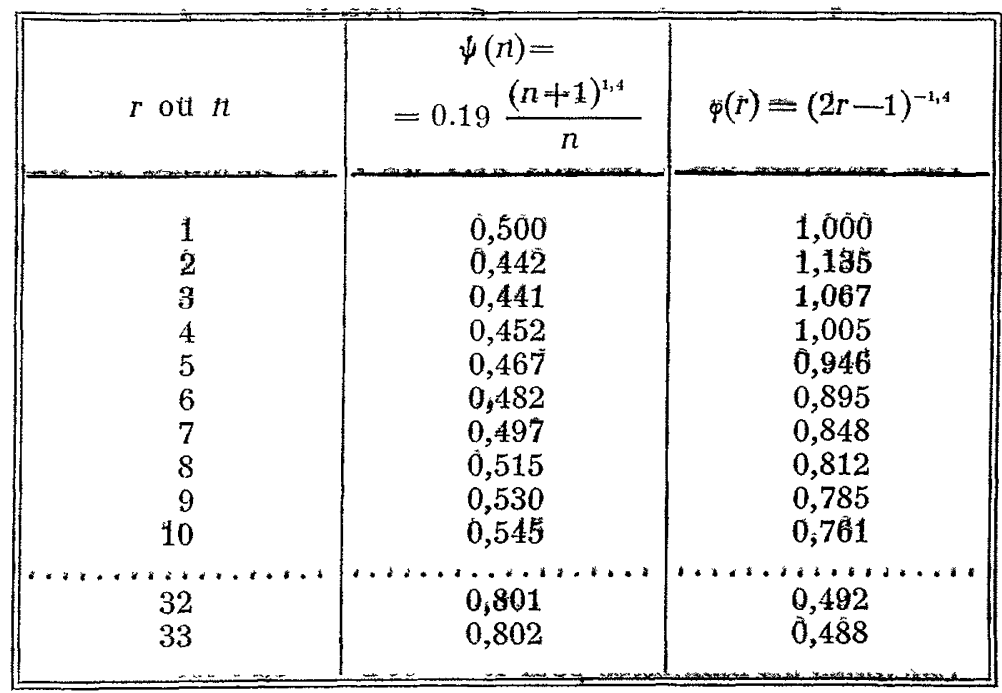

La fonction $\psi(n)$ devient minimum pour $n=2,5$ et sa valeur est 0,439 ; elle est done minimum pour la valeur de $n$ entre 2 et 3 et par la suite croît avec $n$.

Quant à la fonction $\varphi(r)$ analytiquement, elle s'annule pour $r=0,5$ et atteint sa valeur mâximum égale à 1,14 pour $r=1,75$, la valeur de la fonction devient égale à l'unité pour $r=1$ et $r=\sim 4$; pratiquement, elle varie de 1 à un maximum de 1,135 pour des valeurs de $r$ variant de 1 à 2 et décroît ensuite progressivement lorsque $r$ aügmente.

Pour rendre plus évidente la méthode et le principe sur lequel 
elle se base, on fera quelques applications. Lorsque $n=2$, $r$ ne pouvant prendre que les valeurs 1 et 2 , on aura alors :

$$
\begin{aligned}
& \downarrow_{(2)}=0,442, \quad h_{1}=\frac{1}{4}, \quad h_{2}=\frac{3}{4} \mathrm{H} \\
& \varphi_{(1)}=1, \quad \varphi_{(2)}=1,135 \\
& d_{1}^{7}=4 \times 0,442 \times 1 \times \mathrm{D}^{7}=1,76 \mathrm{D}^{7} \\
& d_{9}{ }^{7}=1,33 \times 0,442 \times 1,135 \times \mathrm{D}^{7}=0,664 \mathrm{D}^{7}
\end{aligned}
$$

d'où :

$$
d_{1}=1,08 \mathrm{D} \quad \text { et } \quad d_{2}=0,94 \mathrm{D}
$$

Résultats qui sont en accord parfait avec ceux que l'on obtiendra par l'expression :

$$
d_{\mathrm{r}}=0,87 \mathrm{D}(n+1)^{0,2} r^{-0,2}
$$

En faisant des calculs analogues pour des valeurs de $n=3$

\begin{tabular}{|c|c|}
\hline $\begin{array}{c}\text { Nombre } \\
\text { de tronçons }\end{array}$ & Diamètres \\
\hline 2 & $\begin{array}{l}\mathrm{d}_{1}=1,08 \mathrm{D} \\
\mathrm{d}_{2}=0,94 \mathrm{D}\end{array}$ \\
\hline 3 & $\begin{array}{l}\mathrm{d}_{1}=1,15 \mathrm{D} \\
\mathrm{d}_{2}=0, \mathrm{D} \\
\mathrm{d}_{3}=0,92 \mathrm{D}\end{array}$ \\
\hline 4 & $\begin{array}{l}\mathrm{d}_{1}=1,20 \mathrm{D} \\
\mathrm{d}_{2}=1,04 \mathrm{D} \\
\mathrm{d}_{3}=0,96 \mathrm{D} \\
\mathrm{d}_{4}=0,91 \mathrm{D}\end{array}$ \\
\hline
\end{tabular}
et $n=4$ et en groupant les résultats obtenus, on a érigé le tableau suivant :

Diamètres des conduites rectilignes verticales ou inclinées, coniques, en fonction du diamètre de la conduite correspondante à section constante:

Des résultats ausci parfaits avec les deux formules ne sont plus obtenus lorsque la charge sur la prise de la conduite n'est plus négligeable, tout au moins par rapport à la charge moyenne sur le premier tronçon ou sur la partie à épaisseur constante.

En effet, dans ce cas on a :

$$
h_{\mathrm{r}}=h_{\mathrm{o}}+\frac{\mathrm{H}}{n}\left(r-\frac{1}{2}\right)
$$

Exemple numérique. - Pour l'exemple cité dans la figure 1, dont la charge $h_{0}$ est de $20 \mathrm{~m}$. se rapportant à la pression maximum de la première dénivellation.

Calculons les diamètres pour $n=4 ; D=0,77 \mathrm{~m}$.

Chaque tronçon a une longueur de $247 \mathrm{~m}$. 50 et le premier d'entre eux comprend la partie de $100 \mathrm{~m}$. de longueur à épaisseur constante, celle de $140 \mathrm{~m}$. qui reste de la première dénivellation et $7 \mathrm{~m} .50$ de la seconde.

En employant l'expression :

on a :

$$
h_{\mathrm{r}} d_{\mathrm{r}}^{7}=\mathrm{H} \mathrm{D}^{7} \psi(n) \varphi(r)
$$

$$
\mathrm{H} \mathrm{D}^{7}=72,80 . \quad \psi_{(4)}=0,452, \quad h_{\mathrm{r}} d_{\mathrm{r}}^{7}=32,9 \stackrel{\text { }}{ }(r)
$$

et en introduisant pour les valeurs de $h$ les vraies pressions moyennes des différenls tronçons, on obtient les résultals sui. vants :

$$
d_{1}=0,88 ; \quad d_{2}=0,80 ; \quad d_{3}=0,73 ; \quad d_{k}=0,69
$$

En employant la formule :

$$
d_{\mathrm{r}}=0,87 \mathrm{D}(n+1)^{0,2} r-1,2
$$

on aboutit aux résultats :

$$
d_{1}=0,92 ; \quad d_{2}=0,80 ; \quad d_{3}=0,74 ; \quad d_{4}=0,70
$$

Ie premier tronçon, dans les deux cas, doit encore être subdivisé pour tenir compte de la première partie à épaisseur constante. En supposant la subdivision en deux parties, on obtient respec tivement les diamètres :

$$
\begin{array}{ll}
d_{1}^{\prime}=0,95 & d_{1}{ }^{\prime \prime}=0,83 \\
d_{1}^{\prime}=0,99 & d_{1}^{\prime \prime}=0 \text { ' } 87
\end{array}
$$

A partir du deuxième tronçon, comprenant le reste de la deuxième dénivellation et une partie de la troisième, les résul. tats obtenus par les deux formules sont pratiquement parfaitement concordants.

\section{II. - Critérium économique pour la détermination des pertes de charge dues aux frottements}

La perte de charge totale $Y$ aussi bien que la perte de charge spécifique $y$ doivent être déterminées en se basant sur de nom. breuses considérations d'ordre technique et économique.

Dans ma première étude, je notifiais que la perte de charge ${ }^{\prime}$ devait être prise égale à un pourcentage déterminé de la chute totale, ce qui correspond au critérium économique de fixer à priori le rendement de la conduite en considérant cette dernière comme un tout indépendant.

En effet, lorsqu'on attribue à $Y$, par exemple, la valeur : $5 \% H$, cela équivaut à admettre un rendement de $95 \%$ pour la conduite. Nous développerons plus particulièrement cette conception par la suite.

Le rendement global d'une installation hydro-électrique est. au point de vue technique, une fonction directe de plusieurs rendements partiels ; quant au point de vue économique, le render ment cherché dépend des résultats que l'on obtient avec l'entreprise envisagée. L'étude préliminaire d'une entreprise hydro. électrique doit tenir compte aussi bien des coefficients techniques que de ceux économiques qui se présentent automatiquement en cours d'étude. Plus on s'avance vers la fin plus se précisent les buts et les moyens de l'entreprise projetée.

Si l'énergie doit être vendue à une industrie ou à une localité d'un faible pouvoir d'achat, on devra, sans autre, consentir à une augmentation de la perte de charge $Y$, afin de pouvoir réduire les frais d'installation de la conduite, ainsi que ceux de toutes les parties de l'installation du centre pro* ducteur qui en sont susceptibles; ce critérium peut s'étendre aussi aux ouvrages concernant le transport et la distribution de l'énergie.

D'autres conceptions, inspirées par des considérations écono. miques ont été proposées soit pour la détermination de la perte de charge $Y$ à attribuer à une conduite avec diamètre conjs tant $D$ ou avec des diamètres progressivement variables $d_{i}$ que pour la détermination directe de son diamètre $D$ ou pour la succession décroissante des diamètres $d$. 
C'est ainsi qu'en 1897, le professeur Zunini dans son projet d'aménagement de l'Orba, a développé et suivi la conception dile : " du maximum d'avantages".

Un autre critérium a été défini " critérium du bénéfice annuel maximum ", il prend en considération surtout la valeur des capitaux inveslis, ainsi qu'un grand nombre de coefficients d'exploilation : Prix de revient et prix de vente du kilowattheure, taux d'intérêt et d'amortissement, dépenses annuelles d'exploitation el d'entretien. Les développements analytiques s'inspirant de cette idée générale ont élé très nombreux, mais la conclusion finale a loujours été exprimée par une équation hyperbolique de $7^{\text {me }}$ degré.

Dans ce qui va suivre, on va essayer de déterminer la valeur de $Y$ ou bien de $D$ en ne considérant pas uniquement les principes économiques déjà exposés : $1^{\circ}$ Valeur minimum du capital (installation et perte d'énergie); $2^{\circ}$ considération essenlielle du rendement de la conduite; Mais il ne sera pris en considération, autant que possible, que la dépense minimum des frais d'installation.

Dans tous les cas, la détermination de $Y$ et de $D$ doit servir pour le calcul d'évaluation des valeurs à donner aux différents diamèlres $d$ de la conduite conique. Les avantages des canalisations à diamètres décroissants sont, en effet, tellement importants qu'on ne peut ne pas conseiller dans chaque cas une canalisation à section progressivement décroissante en remplacement d'une canalisation à section constante.

Dans la dernière partie de cette étude, il sera présenté de la façon la plus complète un nouveau type de conduite conique avec diamètre constant pour chaque dénivellation; les pertes de charge spécifique croissant en proportions arithmétiques depuis le tronçon initial jusqu'à la dénivellation finale. La conduite sera donc constituée de tronçons de longueur variable dont le nombre sera représenté par celui des dénivellations plus un. Le développement analytique du critérium susindiqué sera complété par des exemples pratiques d'applications numériques.

Toutefois, on a cherché d'atteindre les résultats pratiques désirés en se servant de procédés aussi simples que possible. A cet effet, et pour conserver la simplicité d'expression, on a généralement adopté des équations algébriques entières, avec exposants décimaux positifs ou négatifs. Dans le même but, on a conservé la formule connue de la perte de charge :

avec $K=0,0025$.

$$
\mathrm{Y}=\mathrm{I}^{r} \mathrm{~L} \mathrm{Q}^{2} \mathrm{D}-5
$$

Les exposants aussi bien que les coefficients dans les valeurs adoptées doivent être considérés comme des symboles et pouvoir facilement être remplacés suivant le cas auquel ils se rapportent par d'autres cocfficients de pertes par frottement et d'autres exposants.

Signalons en passant qu'à ce type de formule, avec ce coefficient de perte de charge, peuvent aussi être réduites les formules exponentielles modernes.

C'est ainsi que la formule de Williams-Hazen pour conduites en acier rivées

$$
\mathrm{V}=76,5\left(\frac{\mathrm{D}}{4}\right)^{0,63} \mathrm{~J}^{0,54}
$$

se réduit à cette autre :

$$
\mathrm{Y}=0,0026 \mathrm{~L} \mathrm{Q}^{1,85} \mathrm{D}^{-4,86}
$$

De même la formule de Scobey pour conduites rivées :

$$
1000 i=a v^{1,9} \mathrm{D}^{-1,1}
$$

avec $a=1.35$ (tôles épaisses), peut être transformée comme suit :

$$
\mathrm{Y}=0,0022 \mathrm{~L} \mathrm{Q}^{1,9} \mathrm{D}^{-4,9}
$$

\section{A) Capital minimum correspondant aux frais d'installation et aux pertes d'énergie}

La conception du coût minimum, intégral et réel, d'une canalisation, doit être toujours rapportée à la longueur totale de la conduite.

Détermination directe des pertes. - La perte de charge de $Y$ mètres correspond à une perte de puissance exprimée en kilowatts :

$$
W=\frac{1000 Q Y}{102}=\sim 10 Q Y
$$

et à une perte annuelle financière :

où :

$$
\mathrm{S}=10 \mathrm{Q} \text { Y } \mathrm{R}
$$

$\eta$ est le rendement général de l'installation considéré depuis la Chambre de mise en charge jusqu'au départ de l'énergie de la centrale et :

$R_{1}$ est le prix de vente du kilowatt-an au départ sus-indiqué.

Dans ce calcul, on doit introduire la valeur du rendement $\eta$ car la perte de charge $Y$ réduit la puissance disponible non seulement dans la conduite, mais dans tout le système de machine de transformation de l'énergie hydro-électrique.

A l'annualité $S$ qui vient d'être définie, correspond une somme, le capital $S_{\text {a }}$ que l'on obtient en multipliant la valeur $S$ par le coefficient de capitalisation.

Pour une concession d'une durée de 60 ans et avec un taux d'intérêt de $6,5 \%$ le coefficient de capitalisation devient égal à 15 .

Il en résulte :

$$
S_{\mathrm{a}}=150 r_{\mathrm{i}} \mathrm{YQR}
$$

On peut donc conclure que le coût réel de la canalisation sera obtenu en ajoutant à la somme correspondant aux frais immédiats d'installation la somme $S a$ correspondant aux kiloxatts perdus.

En indiquant par $\mathrm{P}$ le poids en kilos de l'acier employé, dans la construction de la conduite, et par $C$ le coût total réel et par $a_{1}$ le prix du kilo d'acier, $\mathrm{y}$ compris tous les frais accessoires de fabrication :

$$
\mathrm{C}=c_{1} \mathrm{P}+150 \mathrm{Q} \mathrm{Y} \eta_{\mathrm{R}} \mathrm{R}
$$

Le poids $P$ peut être exprimé en fonction de $D^{2}$ au moyen des formules déjà données

On peut donc écrire :

$$
\mathrm{P}=\mathrm{Z} \mathrm{D}^{2}
$$

En posant :

$$
Z=2 \Sigma h_{\mathrm{mr}} l_{\mathrm{r}}
$$

et puisqüe :

$$
\mathrm{Y}=\mathrm{KL} \mathrm{Q}^{2} \mathrm{D}-5
$$

d'où l'on obtient :

$$
\mathrm{D}=\mathrm{K}^{0,2} \mathrm{~L}^{0,2} \mathrm{Q}^{0,4} \mathrm{Y}^{-0,2}
$$

L'expression de $C$ prendra la forme suivante :

$$
\mathrm{C}=c_{1} Z \mathrm{~K}^{0,4} \mathrm{~L}^{0,4} \mathrm{Q}^{0,8} \mathrm{Y}^{-0,4}+150 \eta \mathrm{Y} \mathrm{QR}
$$

En donnant à $c_{1}$ la valeur habituelle $c_{1}=3,50$ lires, et à $K$ sa valeur, $K=0,0025=25,10^{-4}$; d'où $K^{0,4}=0,09$ et à 
$\eta$ une valeur thoyentle admissible $\eta=0,80$, on aura en détinitive :

$$
\mathrm{C}=0,315 \mathrm{ZL} \mathrm{L}^{0,4} \mathrm{Q}^{0,8} \mathrm{Y}-0,1+120 \mathrm{Y} \mathrm{QR}
$$

10 Perte totale. - Les dérivées successives de $C$ en fonction de $Y$ sont :

$$
\left\{\begin{array}{l}
\mathrm{C}^{\prime}=0,126 \mathrm{ZL} \mathrm{L}^{0,4} \mathrm{Q}^{0,8} \mathrm{Y}-1,4+120 \mathrm{QR} \\
\mathrm{C}^{\prime \prime}=0,1764 \mathrm{Z} \mathrm{L}^{0,4} \mathrm{Q}^{0,8} \mathrm{Y}-\varepsilon, 4
\end{array}\right.
$$

En égalisañt l'expresssion (a) à zéro et en la trânsformant, on âbơtutit facilement à :

$$
\mathrm{Y}^{1,4} \equiv 0,00105 \mathrm{Z} \mathbf{L}^{0,4} \dot{Q}^{-0,2} \mathbf{R}-1
$$

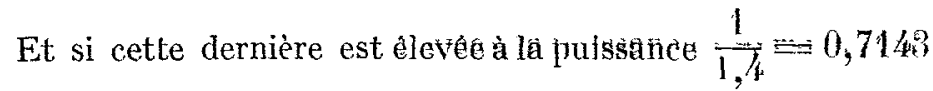
on obtiendra l'expression demandée :

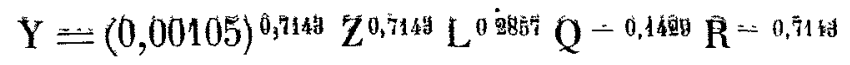

dans laquelle le coefficient numérique est 0,00745 .

On peut done écrire :

$$
\mathrm{Y}=0,00745\left(\frac{\mathrm{Z}}{\mathrm{R}}\right)^{0,7143}\left(\frac{\mathrm{L}^{2}}{\mathrm{Q}}\right)^{0,1429}
$$

Cette valeur de $\Psi$ rend la dérivée seconde de $C$ positive et minimum la fonction exprimée par la relation (7).

La détermination de la valeur minimum de l'expjossion (7) est aisée.

Ẻn divisànt (8) pâr $\left(7^{\prime}\right)$, ôn ổbtieñ :

$$
\mathrm{Y}^{-0,4}=(0,001 \mathrm{Z})^{-0,2857} \mathrm{~L}^{-0,114} \mathrm{Q}^{0,057} \mathrm{R}^{0,2857}
$$

et en remplaçant dans (7) en même temps que dâns (8) les valeurs trouvées, on aủrà :

$$
\dot{\mathrm{C}}_{\mathrm{mm}}=(2,97+0,89) \mathrm{A}=3,16 \mathrm{~A}
$$

ãyanit posé.

$$
A=Z^{0,7143} L^{0.2857} Q^{0,8571} R^{0.2857}
$$

Lè terthé 2,27 dủ coefficient nuthérique dẻ la fonction dè $C$

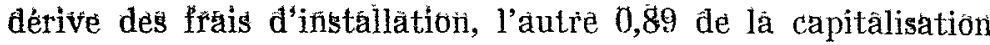
des pertes. En concluant le coût réel minimum d'uné cànalisation, comme nous l'indiquent les résultats obtenus, est composé de deux parties : $72 \%$ attribuables aux frais d'installa-

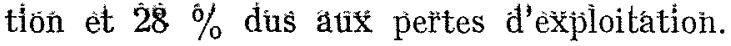

En considérant l'expression de A o déduit que le coût minimum d'une conduite, calculé suivant les cońnidérâtions ádmises, dépend principalement du débit (exposant $=0,86$ ), ensuite de la chute envisagée (exposant de $Z=0,71$ ) et en dernier lleu et dans les mêmes propơrtióńns de la longueur totale de la conduite et du prix de vente du kilowatt-an (exposants dè $L$ et de $R=\sim 0,29$ ).

On en déduit aussi que toute variation de $\mathrm{A}$ influe proportionnellement tant sur les premiers frais d'installation que sur la capitalisation des pertes par frottement.

Pour une conduite déterminée et en exploitalion, la valeur de $A$ varie uniquement avec celle de $R$, cela a une grande importance dans la prévision du prix de vente du kilowatl=an.

En refaisant les mêmes dâleuls pour l'expression généralo de $C$, sans les simplificalions numétiques prétédenimenl envisagées pour $c_{1}, K$ et $r_{1}$ l'expression de (8) sera :

$$
\mathbf{Y}=\left(0,0027 \frac{c_{1}}{r_{1}}\right)^{0,7133}\left(\frac{Z}{R}\right)^{0,7143}\left(\frac{K^{2} L_{2}^{2}}{Q}\right)^{0,1429}
$$

$2^{6}$ Exemple numérique. - - Pour mieux illustrer les forthules précitées, il convient de reprendre le cảs numérique déjà envl: sagé dans ma publication précèdente.

La conduite était caractérísée pâr le profill représenlé à la fig. 1, où : $\dot{H}=455 \mathrm{~m} . \mathrm{L}=990 \mathrm{~m}$., $Q=1 \mathrm{~m}^{3} / \mathrm{sec} ., Y=2 \%$ de $\mathrm{H}=9,10 \mathrm{~m} . ; y=0,00919 \mathrm{~m}$. par mètre courant ct on a vait àtribùe à $\mathrm{K}$ la valèur $: K=25.10^{-4}$.

La conduite fut ealculée avec diamètre constant et avec des diamétres progressivement varlables.

Dans le premier cas, en employant la formule $(5)$, on a :

$$
0,00919=-5.10=4 \times 1 \times \mathrm{D}=5
$$

et on a obtenu : $D=0,77$ et $P=315$ tonnes, La valeur de $P$ a été obtenue au moyen d'un développement des tôles constituant les divers tronçons de lá conduite.

Dáns le secơnd càs, les résultats furent les suivânts :

Diamètres vảriant dê 1,36 nh. à $0,68 \mathrm{~m}$. èt le pởds de lá cờiduitè $P^{\prime}=304$ tônnès, ce der̉nier étant câlcủlé cómñe jprécéć

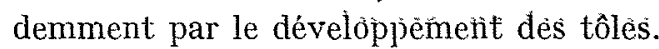

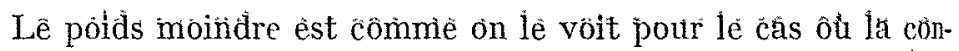

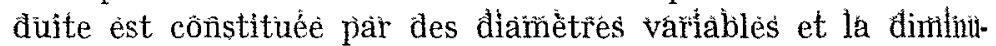
tín du poìds est de 11 tơñiés sur 315 , đônồ de $3,5 \%$.

Appliquons à ce même exemple les annotations et les observations établies en dernier lieu.

Le tèrme en $\%$, relätif à la premièrè dénivellation qui comprend le tionçon initial, en tenant compte que cetle partie est incli née de $30^{\circ}$ sera exprimé par :

$z_{1}=140 \times 100+\frac{19600-4900}{11,5}=14.900+29.400=43.400$

Le premier terme qui, d'ailleurs, n'est pas fractionnaire, se rapporte au tronçon á ćpaisseur constainte: il est alors plus indiqué de l'exprimer pär $z_{4}$; de là : $\ddot{z}_{\bar{\theta}}=14.000$ et $z=29.400$.

Le terme relatif à la quâtrième dénivivellation horizoritale est :

$$
z_{4}=2 \times 455 \times 150=136.500
$$

Les termes des deux dénivellations intermédiaires peuvent se déduire de l'expression générale $z_{\mathrm{r}}=2 h_{\mathrm{mr}} l_{\mathrm{r}}$

On obtient :

$$
\begin{array}{lll}
\sin \alpha_{2}=0,30 & \text { et } & z_{2}=49.750 \\
\sin x_{3}=0,60 & & z_{3}=288.000
\end{array}
$$

Il s'en suit que :

ế :

$$
Z=z_{1}+z_{2}+z_{3}+z_{1}=516.650
$$

$$
P=Z D^{2}=510.650 \times(0,77)^{2}=306.373 \text { tònnès }
$$

Résultat très peu différént de celưi óbtenu par le calcul direct.

Cette différence dérive du fait que l'épaisseur de la conduite ne varie pas d'une façon conlinue et théorique comme l'admet tent les formules, mais; au contraire, d'une facon discontinue d'après les exigences de fabrication du mélal employé. Mais on peut tout de même, d'après les résultats obtenus, affirmer que le procédé indiqué joour exprimer lé poids $P$ en fonction 
du diamètre $\left(D^{2}\right)$ est d'une approximation pratique largement suffisante.

Voyons quels seront les résultats dans le cas envisagé à propos de $R$.

La formule (8) dans le cas qui nous intéresse, et pour les valeurs mises en jeu $\left(L=990 ; Q=1 \mathrm{~m}^{3} / \mathrm{sec}\right.$, ainsi que pour $Z$ qui a déjà été calculé $Z=516650$ ) sera :

$$
\mathrm{Y} \mathrm{R}^{0,7143}=623
$$

el puisque $Y=9,10 \mathrm{~m}$., on en déduil :

$$
\mathrm{R}=371 \text { lires. }
$$

La valeur de $R$ peut être retenue comme produit des heures de fonctionnement pendant une année par le prix du kilowattheure au déparl de la centrale; pour une valeur déterminée de $R$, no oblient un nombre de groupes de ces valeurs (groupes de deux valeurs) théoriquement infini et pratiquement très grand. Lorsqu'il s'agit de centrale hydroélectrique fonctionnant pour une industrie bien déterminée, $R$ est beaucoup plus facle à calculer, en se rapportant à la valeur de la production industrielle par kilowatt-an, plutôt que comme produit d'un certain nombre d'heures par un autre de centimes.

A celle valeur de $R=371$ lires, correspondent aussi deux autres valeurs qui peuvent être considérées comme valeurs limites : $1^{\circ} \mathrm{En}$ supposant qu'il s'agisse de fournir l'énergie électrique à une importante industrie à consommation très conséquente, cas pour lequel on serait obligé de baisser le prix du kilowatt-heure à 5 centimes, le nombre d'heures qui y correspond devra être de l'ordre de $7.400 ; 2^{\circ}$ Si le consommateur est une industrie riche, pour laquelle on puisse porter le prix $d u$ kilowatt-heure à 20 centimes, l'installation pourra ne fonctionner que 1850 heures seulement.

En accouplant les chiffres représentant les prix minima el maxima et les nombres d'heures de fonctionnement, on obtiendrait respectivement : $R=92,50$ lires et $R=1.480$ lires, représentant les valeurs limites entre lesquelles peuvent varier $R$ et par conséquent $D$, la puissance de la centrale, etc.

Pour aboutir au coût minimum, en tenant compte de la dépense d'installation et de la capitalisation des pertes, il faut calculer la valeur de A. Pour les valeurs susindiquées de $Z, L$, $Q, R$, les termes respectifs sont $12050 ; 7,17 ; 1 ; 5,41$ et leur produit nous donnera :

$$
\mathrm{A}=468.500
$$

On constate facilement l'énorme influence du terme $Z$ due aux valeurs relativement élevées de la chute et de la longueur de la canalisation.

Le facteur minimum correspondant au débit sera :

$\mathrm{C}_{\text {n!n }}=(2,37+0,89) \times 468.500=1.063 .495$ lires +416.965 lires

La première partie de la somme représente les frais d'installation et la seconde partie la capitalisation des pertes dans les conditions admises. Le coût réel et total est :

$$
\mathrm{C}_{\mathrm{mm}}=1.480 .460 \text { lires }=3,16 \mathrm{~A} \text {. }
$$

Par un calcul approximatif, mais de beaucoup plus rapide, nous aurions pu obtenir les frais d'installation en nous basant sur le poids de la conduite et en l'égalant, comme il a été démontré, au $72 \%$ de $C_{\text {min }}$. On obtiendrait :

$\mathrm{C}_{\min }=(\mathrm{kg} .306 .373 \times 3,50$ lires $): 0,72=1.489 .000$ lires

Du reste, le calcul exact peut être aussi fait, sans déterminer la valeur de $\mathrm{A}$, en appliquant la formule suivante :

$$
\mathrm{C}=c_{1} \mathrm{P}+120 \mathrm{QYR}
$$

Ce qui nous donnerait :

Dépenses d'installation $=306.373 \mathrm{~kg} . \times 3,50$ lires $=1.072 .305$ lir. Capitalisation de $Y=120 \times 1 \times 9,10 \times 371=.405 .132 \mathrm{lir}$.

Coût total réel ................ 1.477.437 lir.

$\mathrm{Si}$, au contraire, nous supposons $R=1.200$ lires, l'expression (8) se rapportant toujours à l'exemple de la fig. 1 et d'un débit $Q=1 \mathrm{~m}^{3} / \mathrm{sec}$. , nous fournit :

$$
\mathrm{Y}=3,94 \mathrm{~m} . ; \quad \mathrm{D}=0,91 \mathrm{~m} . ; \quad \mathrm{P}=426,6 \text { tonnes }
$$

D'où l'expression de $C$ exprimée par :

$$
\begin{aligned}
C & =(426.600 \times 3,50)+120(\times 1 \times 3,9 \times 1.200)= \\
& =1.493 .000 \text { lires }+561.600 \text { lires }=2.054 .700 \text { lires. }
\end{aligned}
$$

Il en résulte qu'en faisant varier le prix de vente du kilowatt-an de 371 lires à 1.200 lires, les frais de première installation auront augmenté d'environ 420.000 lires, c'est-à-dire de $39 \%$ et la capitalisation des pertes augmente aussi d'environ $39 \%$, conformément à ce qui a été déduit au paragraphe précédent.

$3^{\circ}$ Pertes par mètre courant. - Par un procédé analogue à celui qui nous a conduit à l'établissement de l'expression (8), nous pouvons raisonner sur un élément très petit de la conduite. Nous aboutirons évidemment à des formules qui permettent d'établir le calcul des pertes de charge totale $Y$, ainsi que la détermination directe du diamètre ou des diamètres de la conduite.

En nous rapportant à la longueur de un mètre, pour laquelle on a une perte de charge $y$ et conservant à $K$ la valeur attribuée $K=0,0025 ; K^{0, \xi}=0,09, C=3,50$ lires et $\eta=0,80$.

On obtient :

$$
\begin{aligned}
& y=\mathrm{K}^{2} d^{-5} \\
& p=2 h\left(\mathrm{~K} \mathrm{Q}^{2}\right)^{0,4} y^{-0,4} \\
& c=0,63 h \mathrm{Q}^{0,8} y^{-0,4}+120 y \mathrm{QR}
\end{aligned}
$$

Les dérivées première et seconde de $c$ par rapport à $y$ seront exprimées par :

$$
\left\{\begin{array}{l}
c^{\prime}=-0,252 h \mathrm{Q}^{0,8} y^{-1,4}+120 \mathrm{QR} \\
c^{\prime \prime}=0,3528 h \mathrm{Q}^{0,8} y^{-2,4}
\end{array}\right.
$$

En posant $c^{\prime}=0$, en résolvant pour $y^{1,4}$ et en élevant à la puissance $1 / 1,4=0,7143$, nous obtiendrons successivement :

$$
\begin{aligned}
& y^{1,4}=0,002 h \mathrm{Q}^{-0,2} \mathrm{R}^{-1} \\
& y=0,012 h^{0,7143} \mathrm{Q}^{-0,1429} \mathrm{R}^{-0,7143}
\end{aligned}
$$

En faisant le produit de ces deux équations et en prenant l'inverse du résultat ainsi obtenu, nous aurons :

$$
\begin{aligned}
y^{-24} & =\frac{1}{\left(24.10^{-6} h^{1,7143} \mathrm{Q}^{-0,343} \mathrm{R}-1,71 / 3\right.} \\
& =41667 h^{-1,7143} \mathrm{Q}^{0,343} \mathrm{R}^{1,7143}
\end{aligned}
$$

Cetle valeur portée dans l'expression de la dérivée seconde de $c$ rend celle-ci positive.

Il s'en suit qu'en faisant varier, d'après l'expression (8'), la valeur $y$ avec $h$, on obtient les prix minima par unité de longueur de la conduite envisagée.

En faisant le rapport entre ( $\left.7^{\prime \prime}\right)$ et $\left(8^{\prime}\right)$, nous obtenons une expression en $y]^{-0,4}$, or, en remplaçant la valeur de $y^{-0,4}$ 
ainsi obtenue dans l'équation (7'), nous pouvons en tirer certaines considérations d'intérêt :

$$
\begin{aligned}
& y^{-0,4}=6 h^{-0,2857} \mathrm{Q}^{0,057} \mathrm{R}^{0,2857} \\
& \mathrm{C}_{\min }=5,22 h^{0,7143} \mathrm{Q}^{0,857} \mathrm{R}^{0,2857}
\end{aligned}
$$

La différentielle totale de $C_{\text {min }}$, réduite à sa plus simple expression, se présentera sous la forme :

$$
d c_{\min }=1,49(3 d \mathrm{Q}+2,50 d h+d \mathrm{R})
$$

Nous en concluons d'une façon générale que l'influence des valeurs $R, h$ et $Q$ sur le prix de revient d'une conduite, les calculs étant faits d'après les principes économiques et techniques admis, se fait sentir dans les proportions de $1: 2,5: 3$. Conclusion à laquelle nous avions abouti pour le prix (ou le poids) de la conduite tout entière.

$4^{\circ}$ Critique comparative des différentes méthodes pour la détermination du poids d'une conduite. - En remplaçant dans l'expression (6') la valeur déjà déterminée de $y^{-0, \bar{k}}$ et réduisant, nous aboutissons à la valeur suivante :

$$
p=1,08 h^{0,7143} \mathrm{Q}^{0,857} \mathrm{R}^{0,2857}
$$

qui est homogène à la formule exprimant la valeur de $C_{\text {min }}$, résultat auquel il fallait s'attendre.

On commettrait une erreur en voulant déduire le poids de la conduite tout entière par l'emploi de la (9) dans laquelle on remplacerait la valeur de $L$ par $\frac{H}{2}$ et en multipliant le tout par la longueur totale de la conduite $L$.

En remplaçant dans l'équation (9) $h$ par le quotient entre la somme des produits de la longueur par la hauteur moyenne de chaque dénivellation et la longueur totale de la conduite $L$, c'est-à-dire en posant :

$$
h=\frac{\Sigma_{1}^{\mathrm{n}} h_{\mathrm{r}} l_{\mathrm{r}}}{\mathrm{L}}
$$

On obtient le poids par mètre courant de la conduite envisagée, qui, s'il n'est pas très exact, reste toutefois de beaucoup moins erroné de celui qu'on obtiendrait en posant :

$$
h=\frac{\mathrm{H}}{2}
$$

Dans les deux cas pourtant, on devrait introduire un terme correctif pour le tronçon initial.

La valeur exacte de la pression hydrostatique $h_{\mathrm{m}}$ correspondant au poids réel moyen par unité de longueur d'une conduite déterminée, s'obtient en confrontant les équations (1) et (5) puisque l'on doit avoir :

$$
\mathrm{P}=\mathrm{Z} \mathrm{D}^{2}=2 h_{\mathrm{n}} \mathrm{D}^{2} \mathrm{~L}
$$

d'où :

$$
\mathrm{Z}=2 h_{\mathrm{m}} \mathrm{L}
$$

Ce qui nous donne la valeur réelle de $h_{\mathrm{nI}}$.

$$
h_{\mathrm{m}}=\frac{\mathrm{Z}}{2 \mathrm{~L}}
$$

Cette hauleur ainsi définie peut être dénommée " pression moyenne pondérale de la conduite \%. Elle ne dépend que du jrofil de la canalisation qui caractérise les valeurs de $L$ et $Z$.

Pour la fig. 1, au profil donné la valeur de $Z$ étant 516.650 , on en déduit :

$$
h_{\mathrm{m}}=261 \mathrm{~m} \text {. }
$$

Valeur que l'on peul employer pour déterminer les poids des deux conduites déjà envisagées à $D=0,77$ et $D=0,92$.

Dans le tableau ci-dessous, nous avons rapporté les poids totaux de la conduite considérée, le diamètre étant pris égal à $0,77 \mathrm{~m}$. Ces poids ont élé oblenus par les trois méthodes qui viennent d'être exposées et par la mélhode directe, ce qui permet de faire la comparaison entre les résultats obtenus. Ia méthode directe est obtenue par le développement de la tôle qui constitue la conduite.

Les deux premiers poids ont été corrigés pour tenir compte du premier tronçon à diamètre constant dans la première dénivellation.

Des pourcentages marqués dans la troisième colonne, on déduit l'erreur à observer pour les diverses méthodes employées.

Poids en tonne de diverses canalisations de même profil repré senté dans la figure 1 et à diamètre constant:

$$
\begin{gathered}
\mathrm{D}=0,77 ; \mathrm{H}=455 \mathrm{~m} . ; \quad \mathrm{Y}+9,10 \mathrm{~m} . \\
y=0,00919 \mathrm{~m} . ; \mathrm{L}=990 \mathrm{~m} .
\end{gathered}
$$

\begin{tabular}{|c|c|c|}
\hline \multicolumn{1}{|c|}{$\begin{array}{c}\text { en mètres } \\
\text { en }\end{array}$} & P \\
\cline { 2 - 3 }$h=0,5 \mathrm{H}$ & tonnes & $\%$ \\
\hline$h=\left(\frac{\sum_{1}^{\mathrm{n}} h_{r} l_{r}}{L}\right)$ & 272,9 & 86,7 \\
$\mathrm{P}=\mathrm{Z} \mathrm{D}{ }^{2}=2 h_{m} \mathrm{~L}$ & 292,3 & 92,8 \\
Calcul direct & 306,4 & 97,3 \\
\hline
\end{tabular}

b) Détermination directe du diamìtre. - De l'équation :

$$
\mathrm{C}=c_{1} \mathrm{P}+150 \eta_{1} \mathrm{YQR}
$$

et en y remplacant par leur valeur respective : $P=Z D^{2}$, $\eta=0,80$ et $Y$ obtenus de l'équation (6), on aura :

$$
\mathrm{C}=c_{1} \mathrm{ZD}^{2}+120 \mathrm{~K} \mathrm{~L} \mathrm{R} \mathrm{Q} \mathrm{Q}^{3} \mathrm{D}^{-5}
$$

En dérivant par rapport à $D$, en remplaçant par leurs valeurs $c_{1}$ et $K\left(c_{1}=3,50\right.$ lires et $\left.K=0,0025\right)$ en réduisant et en transformant ayant d'abord égalisé à zéro, on obtiendra :

$$
Z \mathrm{D}^{7}=0,214 \mathrm{~L} \mathrm{R} \mathrm{Q}^{3}
$$

qui est l'expression générale qui lie la variation du diamètre au profil de la conduite, lorsqu'on veut adopter la formule (6') pour rendre minimum le capital investi dans l'installation, ainsi que la perte d'énergie.

Etant donné que $Z$ est fonction uniquement du profil de la canalisation, sa valeur peut être calculée avant toute autre quantité caractéristique de la conduite.

Pour l'exemple que nous avons constamment considéré et qui est représenté par la fig. 1 , nous avons : $Z=516.650$; $R=371 ; 4=990 ; Q=1$ et en appliqaant la formule (11), on obtient :

$$
\mathrm{D}^{7}=0,15 \quad \text { d'où } \quad \mathrm{D}=0,765
$$

valeur qui, comme on le voit, est très proche de celle $D=0,77$ correspondant aux données techniques habituellement considérées pour la conduite en question et à une valeur de $R=371$.

La loi de variation du diamètre dans une conduite conique s'obtient en égalisant entre elles les deux équations connues : $\left(8^{\prime}\right)$ et $\left(5^{\prime}\right)$, ce qui donnera :

$$
0,0025 \mathrm{Q}^{2} d^{-5}=0,012 h^{0,7133} \mathrm{Q}^{-0,143} \mathrm{R}-0,7143
$$


en élevant celle-ci à la puissance 1,4, qui simplifiée et transformée, nous donne la suivante :

$$
h d^{7}=0,1112 \mathrm{R} \mathrm{Q}^{3}
$$

L'identification entre les deux équations (11) et (12) redonne la relation bien connue :

$$
Z=\sim 2 h_{\mathrm{m}} \mathrm{L}=1980 h_{\mathrm{m}}
$$

dans laquelle $\mathrm{hm}$ représente la quantité définie sous le nom de "pression hydrostatique moyenne".

l'our une conduite à diamètres variables, nous déduirons de l'équation (12), toujours pour les mêmes conditions $Q=1$ el $R=371$, l'équation :

$$
h d^{7}=4
$$

dans laquelle, l'ayant réduite sous forme logarithmique et en remplaçant $h$ successivement par les profondeurs moyennes des deux tronçons de la première dénivellation et des trois autres dénivellations, nous obtiendrons les résultats suivants (résultats qui ont été pratiquement arrondis au centimètre près).

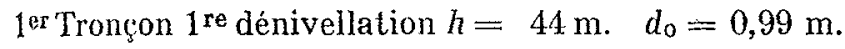

$2^{\mathrm{e}}-1^{\mathrm{re}}-h=105 \mathrm{~m} . d_{1}=0,87 \mathrm{~m}$.

- $\quad 2^{\mathrm{e}} \quad-\quad h=162,50 \quad d_{2}=0,82 \mathrm{~m}$.

$3^{\mathrm{e}} \quad \cdots \quad h=320 \mathrm{~m} . d_{3}=0,75 \mathrm{~m}$.

$4 \mathrm{e} \quad-\quad h=455 \mathrm{~m} . d_{\mathrm{d}}=0,71 \mathrm{~m}$.

L'expression (12) nous donnera, pour la valeur réelle $h$ moyenne pondérale, déjà calculée en mètres et égale à 261 mètres :

$$
Z=516.780
$$

Valeur très proche de celle qui a été déterminée directement.

Pour l'autre conduite, qui diffère de la précédente uniquement par la valeur de $R$, dans ce cas, nous adoptons pour $R$ la valeur de 1.200 lires, et en poursuivant avec le même raisonnement que précédemment, on obtient :

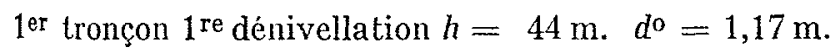

$2^{\mathrm{e}} \quad-1^{\mathrm{re}} \quad-\quad h=105 \mathrm{~m} . d_{1}=1,04 \mathrm{~m}$.

$$
\begin{array}{llll}
- & 2^{\mathrm{e}}- & & h=162,50 d_{2}=0,99 \mathrm{~m} . \\
- & 3^{\mathrm{e}}- & h=320 \mathrm{~m} . d_{\mathrm{a}}=0,88 \mathrm{~m} . \\
- & 4^{\mathrm{e}}- & h=455 \mathrm{~m} . d_{4}=0,84 \mathrm{~m} .
\end{array}
$$

Les poids de ces deux dernières canalisations à diamètres variables diminuant du haut en bas peuvent se calculer simultanément et très facilement comme somme des produits des carrés des diamètres par la valeur de $Z$ qui a été déjà déterminée.

On obtient respectivement 304,1 tonnes pour la conduite avec $R=371$; et 418,5 tonnes pour l'autre conduite avec $R=1.200$.

En concluant : Pour une conduite à section constante, on doit appliquer la formule (11) pour en calculer le diamètre; pour une conduite à section variable, les diamètres successifs doivent être déterminés au moyen de la formule (12).

\section{B) Capital minimum investi}

Le procédé qui sera développé dans ce qui suit découle de celui bien connu se basant sur la puissance hydraulique maximum d'une masse de liquide s'écoulant dans une conduite. Ce procédé est indépendant des prévisions économiques d'exploitation. Il présente aussi de l'intérêt du fait qu'il permet d'étudier les vitesses admissibles dans une conduite et d'en déduire la vitesse maximum admissible.

Pour un exposé rapide et clair de la méthode, il faut envisager avant tout les lois de variation de la puissance utile d'une con- duite pendant la variation totale du débit de zéro à sa valeur maximum théoriquement admissible.

On considèrera les régimes de charge sous les aspects que prennent les différents facteurs : puissances (totale utile et perdue); vitesses de l'eau dans la conduite; rendements successifs correspondant, etc.

a) Puissance utile $\left(P_{\mathrm{u}}\right)$ d'une conduite. - Le débit d'une masse d'eau de $Q \mathrm{~m}^{3} / \mathrm{sec}$. sous une chute de $H$ mètres, s'écoulant dans une conduite de diamètre $D$ et de longueur $L$, avec une perte de charge totale $Y$ dont le coefficient des pertes par frottement est $K$, fournira une puissance utile exprimée en kilowatts, qui sera pratiquement déterminée par l'équation suivante :

$$
\mathrm{P}_{\mathrm{u}}=\mathrm{P}_{\mathrm{t}}-\mathrm{P}_{\mathrm{p}}=10 \mathrm{Q}(\mathrm{H}-\mathrm{Y})
$$

dans laquelle la puissance théorique est représentée par $P_{\mathrm{t}}=$ $10 Q \mathrm{H}$ et la puissance perdue par $P_{\mathrm{p}}=10 Q \mathrm{Y}$. En y remplaçant $Y$ par sa valeur déterminée au moyen de l'équation (5), nous aurons :

$$
P_{u}=10 Q\left(H-K L Q^{2} D^{-5}\right)
$$

De cette dernière équation de forme générale, on constate que la puissance utile $P u$ s'annule, soit pour $Q=o$ soit pour la valeur de $H=K L Q^{2} D^{-3}$, donc pour une valeur de $Q$ qui rend la perte de charge $Y$, due aux frottements, égale à la chute disponible $H$, cette valeur de $Q$ est donc la valeur maximum possible du débit dans la conduite considérée.:

$$
\mathrm{Q}_{\max }=\sqrt{\frac{\overline{\mathrm{HD}^{5}}}{\mathrm{KL}}}=\mathrm{M}
$$

les dérivées première et seconde de l'expression (13) en fonction de $Q$ sont :

$$
\frac{d \mathrm{P}_{\mathrm{u}}}{d \mathrm{Q}}=10 \mathrm{H}-30 \mathrm{KL} \mathrm{Q}^{2} \mathrm{D}^{-5}+10\left(\mathrm{H}-3 \frac{\mathrm{Q}^{2}}{\mathrm{M}^{2}}\right)
$$

et :

$$
\frac{d^{2} \mathrm{Pu}_{\mathrm{u}}}{d \mathrm{Q}^{2}}=-60 \mathrm{KLQD}-\bar{s}
$$

En égalisant à zéro la dérivée première el en résolvant l'équation ainsi obtenue par rapport à $Q$ on en déduira la valeur du débit qui correspond à la puissance utile maxima de la conduite envisagée. En effectuant cette opération au moyen du résultat que peut fournir l'équation (14), on obtient :

$$
\mathrm{Q}_{\mathrm{Pu} \max }=\frac{\mathrm{M}}{\sqrt{\overline{3}}}=0,578 \mathrm{M}
$$

Il en résulte que la puissance utile maxima $P u$ max. correspond aux 6,10 environ du débit maximum théorique de la conduite. En remplacant dans l'équation (13) la valeur définie par la (15), on obtient l'expression de cette puissarce utile maxima et qui est :

$$
\mathrm{P}_{\mathrm{u} \max }=3,85 \mathrm{H} \mathrm{M}
$$

Tous ces résultats permettent le tracé du diagramme de la puissance utile en fonction du débit.

Dans la figure 3 , on a choisi comme unité de débit le débit maximum admissible $M$, comme unité de puissance le produit $H M$, et pour les vitesses $1=M D^{-2}$.

Nous voyons que toutes les quantités représentées dans ce graphique le sont en fonction de $M$ que nous retrouverons d'ailleurs très souvent par la suite, pour cela, et à cause de son 
importance intrinsèque, nous indiquerons cette quantité par les noms " unité caractéristique " ou simplement " caractéristique " de la conduite. Car, en effet, cette quantité jointe au profil d'une conduite nous permet de résoudre en entier le problème que pose une conduite forcée.

Il faut signaler qu'en $M$ est aussi compris le coefficient de frottement $K$ dont l'importance est considérable, surtout lorsqu'il s'agit de conduites très longues et à très grande pression.

Sur le graphique fig. 3 sont représentées les courbes : droite

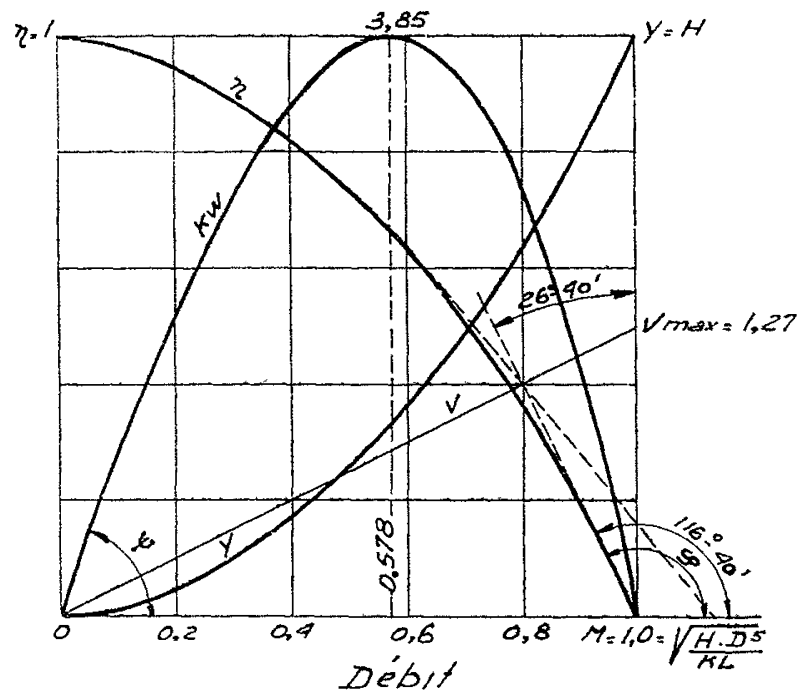

Fig. 3

de vitesses en fonctio: des débits, ainsi que la courbe des rendements. Comme conséquence et à la suite des unités arbitrairement choisies, les diagrammes de la figure 3 sont très clairs, mais assez déformés.

Car si nous avions conservé la même unité de mesure pour les débits et les puissances, la courbe de puissances aurait été beaucoup plus haute. Les angles $\psi$ et $i$ sont proches de $\frac{\pi}{2}$, le premier par défaut et le second par excès, comme on peut facilement s'en rendre compte de l'expression générale de la dérivée première de la puissance utile en fonction des débits. Car pour $Q=o$ et pour $Q=M$, on obtient respectivement :

d'où :

$$
\operatorname{tg} \psi=10 \mathrm{H} \quad \text { et } \quad \operatorname{tg} \varphi=-20 \mathrm{H}
$$

$$
\operatorname{tg} \psi=-0,5 \operatorname{tg} \varphi
$$

Les angles $\because$ et $\psi$ sont d'autant plus grands que la chute $H$ le sera elle aussi, mais même pour des chutes relativement faibles, ces angles restent assez grands, en effet, pour $4=10 \mathrm{~m}$. on aura :

$$
\operatorname{tg} \psi=100 \quad \text { et } \quad \operatorname{tg} \varphi=-200
$$

d'où :

$$
\psi=89025 \quad \text { et } \quad \varphi=90^{\circ} 20^{\prime}
$$

On voit que pour tracer la courbe des $P_{\mathrm{u}}$, les tangentes à l'origine et à la fin de la courbe ne sont pas indispensables puisqu'on peut les retenir pratiquement très voisines des verticales à l'axe des abscisses.

On peut facilement connaitre une autre tangente à la courbe des puissances utiles, celle en son point maximum et qui est parallèle à l'axe des abscisses, car on a :

$$
\mathrm{Q}_{\mathrm{P} \max }=\frac{\mathrm{M}}{\sqrt{\overline{3}}}, \quad \frac{d \mathrm{P}}{d \mathrm{Q}}=0
$$

b) Rendements. - La courbe du rendement ${ }_{1}$ correspond ì l'expression :

$$
r_{1}=\frac{\mathrm{H}-\mathrm{Y}}{\mathrm{H}}=1-\mathrm{KL} \mathrm{Q}^{2} \mathrm{H}^{-1} \mathrm{D}^{-5}=1-\left[\frac{\mathrm{Q}}{\mathrm{M}}\right]^{2}
$$

Le rendement est maximum et égal à l'unité lorsque $Q=0$, tandis qu'il devient nul pour le débit maximum $Q=M$. Pour la puissance utile maximum, le rendement est :

$$
n_{1}=1-\frac{1}{3}=66,7 \%
$$

Que l'on obtient en remplaçant dans l'expression de (17) la valeur du débit correspondant déduil de (15) et dont la valeur est :

$$
Q_{P u \max }=\frac{M}{\sqrt{3}}
$$

Si on exprime la perte de charge $Y$ sous forme de fraction $m$ de $H$, c'est-à-dire si on pose :

$$
\mathrm{Y}=m \mathrm{H}
$$

L'équation (17) prend la forme suivante :

$$
r_{1}=1-m \text { d'où } \quad m=1-r_{1}
$$

ce qui exprime la perte de charge en fonction du rendement.

A la puissance utile maximum $\left(n=\frac{2}{3}\right)$ correspond donc $m=\frac{1}{3}$ et une perte de charge $Y=\frac{\mathrm{H}}{3}$.

De la courbe de rendement, on peut avoir aussi les tangentes, ayant pris comme unité des $r_{i}$ la même longueur représentant l'unité de $Q$, dans l'expression de (17), on aura :

$$
\frac{d \eta}{d Q}=-2 \frac{Q}{M^{2}}=\operatorname{tg} \alpha
$$

étant donné que $M=1$, on aura :

$$
\operatorname{tg} \alpha=-2 Q
$$

Lorsque $Q=0, \operatorname{tg} \alpha=0$ et la tangente est horizontale, tandis que pour $Q=M=1$.

$$
\operatorname{tg} \alpha=-2, \alpha=116^{\circ} 40^{\prime}
$$

et pour le point se rapportant à la puissance utile maxima, pour lequel :

$$
Q=\frac{1}{\sqrt{\overline{3}}}, \quad \alpha=\sim 159^{\circ}
$$

Grâce à ces trois tangentes, on peut tracer à priori le diagramme des rendements sans aucune autre recherche préalable.

Les variations du débit et de la vitesse dans une conduite déterminée sont très fréquentes, soit à cause de la variation de charge du réseau, donc du nombre de groupes hydro-électriques en fonctionnement, soit à cause des variations de débits dues à la disponibilité de l'eau.

Ces variations peuvent donc être très étendues.

Le diagramme des puissances uliles de la figure 3 démontrit qu'il ne convient pas de dépasser les quatre dixièmes environ du débit maximum, car on n'en tire pas un.avantage propol tionnel. On constate aussi que c'est un réel désavantage de dépasser le $58 \%$ du débit maximum, car la puissance utile tout en restant assez élevée diminue très rapidement, aịnsi qué le rendement, Landis que la vitesse augmente de plus en pllst. rapidement. 
De l'examen du diagramme précité, on en déduit aussi que pour les faibles vitesses, on a dés rendements élevés, mais les pussances utiles sont très limitées et, par conséquent, il y a une grande différence entre la puissance normale et la puissance maximum.

On en déduil immédiatement qu'il convient de rapprocher ces deux puissances et pour cela de diminuer le diamètre en augmentant en proportion la vitesse d'écoulement.

c) Exemple numérique. - Une application pratique de ce qui vient d'être exposé, soit sur les formules établies, soit sur les déductions faites des diagrammes qui en dérivent, semble présenter un intérêt très évident.

En nous reportant au cas toujours cité et en conservant toutes les domnées déjà définies, en considérant d'abord $I)=0,77$, on déduil directement des formules précédentes :

$$
\begin{aligned}
& Q_{\max }=7,045 \mathrm{~m}^{3} / \mathrm{sec} \\
& \mathrm{P}_{\max }=12.26 / \mathrm{kW} \\
& Q_{\mathrm{P} \max }=4,046 \mathrm{~m}^{3} / \mathrm{sec} \\
& V_{\max }=15 \mathrm{~m} / \mathrm{sec} \\
& v_{\mathrm{P} \max }=8,65 \mathrm{~m}^{3} / \mathrm{sec}
\end{aligned}
$$

Les conditions de fonctionnement normal de la conduite envisagée dans ma première publication étaient, au contraire, prévues pour un débit de $1 \mathrm{~m}^{3} / \mathrm{sec}$. et une puissance théorique $P_{\mathrm{t}}$ indépendante des pertes dans la conduite de $4.550 \mathrm{~kW}$. avec une vitesse de $2,14 \mathrm{~m} . / \mathrm{sec}$.

En identifiant la puissance théorique maximum $P_{\mathrm{t}}=10 \mathrm{QH}$ à la puissance utile maximum $\mathrm{Pu} \max$, on a une formule qui détermine le diamètre d'une conduite nouvelle pour laquelle les puissances maximum et normale sont très rapprochées.

On a, en effet, si $P_{\mathrm{t}}=\mathrm{P}_{\mathrm{u} \max }$ :

$$
10 \mathrm{QH}=3,85 \mathrm{H} \mathrm{M}
$$

d'où :

$$
\mathrm{D}^{5}=6,67 \frac{\mathrm{KL} \mathrm{Q}}{\mathrm{H}}
$$

et pour la valeur habituelle de $K=25.10^{-4}$ :

$$
\mathrm{D}^{5}=0,017 \frac{\mathrm{L} \cdot \mathrm{Q}^{2}}{\mathrm{H}}
$$

et finalement :

$$
\mathrm{D}=0,44 \sqrt[5]{\frac{\mathrm{LQ}}{\mathrm{H}}}
$$

Pour l'exemple pris en considération, on obtient de cette formule $\mathrm{D}=0,517$ et une section $\mathrm{A}=0,21 \mathrm{~m}^{2}$.

Dans la figure 4 sont tracées les courbes correspondant tant au diamètre 0,77 qu'au diamètre 0,52 .

L'" unité caractéristique " de la nouvelle conduite est évidemment très différente de celle de la conduite avec le diamètre de 0,77 . On obtiendra, en effet, les valeurs suivantes :

$$
\begin{aligned}
& Q_{\max }=2,60 \mathrm{~m}^{3} / \mathrm{sec} .=\mathrm{M}=\sqrt{\frac{\mathrm{HD}^{5}}{\mathrm{KL}}} \\
& \mathrm{P}_{\max }=4550 \mathrm{~kW} \\
& Q_{\mathrm{P} \max }=0,578 \times 2,6=1,5 \mathrm{~m}^{2} / \mathrm{sec} \\
& \mathrm{V}_{\max }=2,60 / 0,21=12,4 \mathrm{~m} / \mathrm{sec} \\
& \mathrm{V}_{\mathrm{P} \max }=1,5 / 0,21=7,14 \mathrm{~m} / \mathrm{sec} .
\end{aligned}
$$

$$
V_{\text {norinal }}=1,00 / 0,21=4,72 \mathrm{~m} / \mathrm{sec} .
$$

vitesse à laquelle correspond une puissance de $3870 \mathrm{~kW}$. et une perte de charge totale de :

$$
Y=65 \mathrm{~m} \text {. }
$$

En observant les valeurs des vitesses qui correspondent aux puissances normales, on peut déduire qu'avec cette méthode de calcul du diamètre, on aboutil à des vitesses inférieures aux maxima admis : de 7 à $8 \mathrm{~m}$. à la seconde.

En outre, en appliquant les formules (5) et (17), on a :

$$
\begin{aligned}
& \mathrm{P}=\mathrm{Z} \mathrm{D}^{2}=516650 \times 0,77=139,4 \text { tonnes } \\
& r_{1}=1-\frac{\mathrm{Y}}{\mathrm{H}}=85,7 \%
\end{aligned}
$$

et finalement :

$$
R=23,7 \text { lires }
$$

que l'on obtient de l'expression :

$$
\mathrm{Y} \mathrm{R}^{0,71: 3}=623
$$

si l'on veut considérer cette conduite dans la catégorie de celles citées dans la Deuxième Partie.

D'après le diagramme des débits utiles dans la figure 3 , on

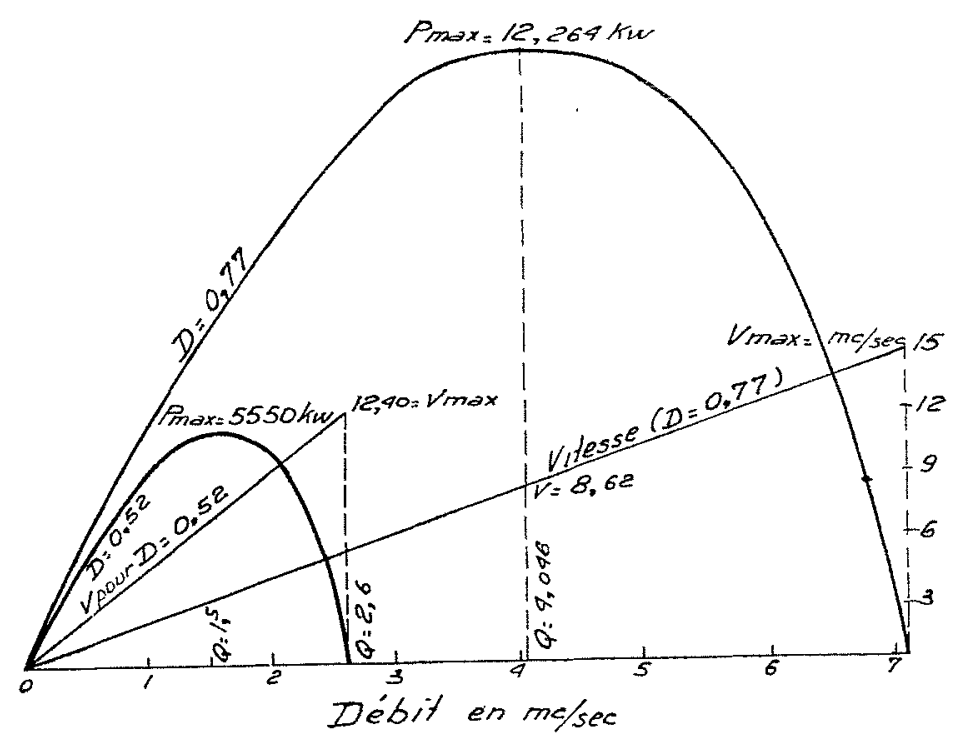

Fig. :

déduit que pour le débit normal $Q=1 \mathrm{~m}^{3} / \mathrm{sec}$., la puissance utile est de $4390 \mathrm{~kW}$., de fàçon que l'on peut facilement obtenir la variation de puissance qui correspond à la variation du diamètre. En passant d'un diamètre de 0,77 à celui de 0,52 , la puissance a diminué de $4390-3870=520 \mathrm{~kW}$.

La diminution de la puissance est așsez importante, mais la diminution du prix global de la conduite nouvelle, aussi bien que de toute l'installation hydro-électrique de la centrale, n'est pas moins sensible.

La diminution du poids de la nouvclle conduite $D=0,52$ par rapport à celle à $D=0,77$ est de $54 \%$ :

$$
306,4-139,4=167 \text { tonnes. }
$$

Le coût réel est intégral $C$ de la conduite devient : 487.900 lires (conduite) +184.800 lires (capitalisation des pertes) $=672.700 \mathrm{lir}$. à confronter avec les résultats obtenus dans les exemples pratiques de la Deuxième Partie.

Mais $R=23,7$ est illusoire : pour $R=371$, on aura :

$$
C=487,900+2.893,800=3.381 .700 \text { lires. }
$$

